\title{
Influência da inversão do diâmetro veia porta/veia esplênica nos resultados do tratamento cirúrgico da hipertensão portal esquistossomótica
}

\section{Influence of the inversion of the portal/splenic vein diameter in the results of the surgical treatment of schistossomotic portal hypertension}

Álvaro Antônio Bandeira Ferraz TCBC-PE1; Josemberg Marins Campos, TCBC-Pe 2; José Guido Corrêa de Araújo Júnior, TCBCPe 2; Márcio Rogério Carneiro de Carvalho 2; João Paulo Ribeiro Neto 2; Edmundo Machado Ferraz TCBC-PE ${ }^{3}$

\section{R E S U M O}

\begin{abstract}
Objetivo: Avaliar a morbidade e a mortalidade no tratamento cirúrgico da hipertensão portal esquistossomótica em pacientes portadores de inversão do diâmetro entre a veia porta e veia esplênica. Métodos: Estudo transversal retrospectivo, de pacientes submetidos ao tratamento cirúrgico da hipertensão no período entre setembro de 1993 e Janeiro de 2004. A população do estudo foi distribuída em dois grupos: a) Inversão - calibre da veia esplênica maior ou igual ao da veia porta) e b) grupo controle (calibre da veia porta maior que o da veia esplênica). Na análise estatística foram utilizados o teste t de student para diferença de médias, quiquadrado para diferença de proporções e o exato de Fisher para amostras reduzidas. Resultados: 169 pacientes foram analisados com seguimento pós-operatório médio de 23,6 meses. 21 pacientes (12,4\%) apresentavam a veia esplênica de igual ou maior calibre que a veia porta (Inversão - grupo de estudo). A média dos diâmetros pré-operatórios das veias porta e esplênica foram, respectivamente, $1,49 / 1,14 \mathrm{~cm}$ no grupo controle, e $0,98 / 1,07 \mathrm{~cm}$ no grupo de inversão. O diâmetro da veia porta foi significativamente maior no grupo controle quando comparado ao grupo de inversão $(p<0,05)$. A presença de varizes de fundo gástrico foi identificada em $33,3 \%$ do grupo de inversão e em 38,5\% dos pacientes do grupo controle. Recidiva hemorrágica pós-operatória ocorreu em $23,1 \%$ dos pacientes do grupo de inversão e em $13,4 \%$ no grupo controle ( $p>0,05)$. Na avaliação pós-operatória com ultrassonografia Doppler de vasos portais, não houve casos de trombose portal no grupo de inversão, e no grupo controle a trombose portal foi identificada em $16,9 \%$ dos pacientes $(p<0,05)$. O óbito ocorreu em um $(4,8 \%)$ paciente do grupo inversão, e a mortalidade foi de $4,1 \%$ no grupo controle $(p>0,05)$. A média do nível sérico de plaquetas foi significativamente menor (65.950 $\mathrm{Imm} \square)$ no grupo de inversão do que no grupo controle $(106.647 \mathrm{Mmm} \square)(p<0,05)$. Conclusão: Os resultados sugerem que a inversão do calibre veia portalesplênica não representa uma contraindicação ao tratamento cirúrgico da hipertensão portal esquistossomótica..
\end{abstract}

Descritores : Esquistossomose. Esplenectomia. Fatores de risco. Veia porta. Hipertensão portal.

\section{INTRODUÇÃO}

A esquistossomose é uma helmintose endêmica em determinadas regiões do território brasileiro, com maior prevalênica na região nordeste e no norte do estado de Minas Gerais, e afeta aproximadamente oito milhões de indivíduos. A forma hepatoesplênica da parasitose é a apresentação mais grave, e ocorre em cerca de $7 \%$ dos infectados. Caracteristicamente, apresenta-se com fibrose hepática periportal e esplenomegalia, induzindo as consequências graves da hipertensão portal.

O tratamento cirúrgico da hipertensão portal esquistossomótica através da esplenectomia com ligadura da veia gástrica esquerda, seguida de esclerose endoscópica pós-operatória, está indicado nos casos associados à hemorragia digestiva alta prévia por ruptura de varizes de esôfago elou gástricas, ou nos casos de hiperesplenismo importante com conseqüências clínicas ao paciente ${ }^{1}$.

Historicamente, as cirurgias de desconexão ázigoportal com esplenectomia tem contribuído para reduzir de forma indiscutível a hemorragia varicosa, principalmente quando associada à intervenções nas varizes, com métodos endoscópicos (ligadura elástica, escleroterapia) e cirúrgicos (ligadura da veia gástrica esquerda, ligadura das varizes de fundo gástrico) ${ }^{1}$.

Pacientes esquistossomóticos podem apresentar deficiências imunológicas importantes, que somadas a um

Estudo realizado no Serviço de Cirurgia Geral do Hospital das Clínicas da Universidade Federal de Pernambuco (SCG HC-UFPE), Recife, Pernambuco, Brasil.

1. Professor Associado e Chefe do Serviço de Cirurgia Geral do Hospital das Clínicas da UFPE- Pernambuco - PE-BR; 2. Professor Substituto do Departamento de Cirurgia da Universidade Federal de Pernambuco (UFPE) - PE-BR; 3. Professor da Pós-Graduação em Cirurgia da Universidade Federal de Pernambuco - PE-BR. 
estado nutricional comprometido e à magnitude do procedimento cirúrgico, conferem um risco elevado para complicações pós-operatórias, particularmente, complicações infecciosas².

$\mathrm{Na}$ tentativa de identificar fatores de risco que possam comprometer a evolução pós-operatória de pacientes submetidos à cirurgias de desconexão e esplenectomia diversos autores tem buscado, de maneira pré-operatória, estabelecer parâmetros que possam aprimorar os resultados tardios neste grupo de pacientes, principalmente no que se refere à recidiva hemorrágica ${ }^{3-22}$

Em alguns pacientes, notamos que havia no estudo com ultrassonografia doppler, feito no pré-operatório, a inversão do calibre portolesplênico (calibre da veia porta $<\mathrm{ou}=$ calibre da veia esplênica). Este achado poderia estar relacionado a uma diminuição brusca da pressão portal após a esplenectomia e consequentemente, aumentar a incidência de trombose da veia porta. Assim como, poderia estar relacionada a uma maior incidência de hiperesplenismo, presença de varizes de fundo gástrico, maior resistência hepática devido ao grau de fibrose periportal. Deste modo, com o objetivo de caracterizar este grupo de pacientes e analisar a influência da inversão do calibre portoesplênica nos resultados do tratamento cirúrgico da hipertensão portal esquistossomótica, é que realizamos este estudo.

\section{MÉTODOS}

Foi realizado um estudo transversal retrospectivo, com revisão de prontuários de pacientes submetidos ao tratamento cirúrgico da hipertensão portal esquistossomótica no período de setembro de 1993 a Janeiro de 2004.

Um total de 169 pacientes operados entre setembro de 1993 e janeiro de 2004 foram incluídos na população do estudo. Parâmetros clínicos, laboratoriais e ultrassonográficos foram registrados e classificados em variáveis contínuas ou categóricas. A população do estudo foi dividida em dois grupos, de acordo com o calibre das veias esplênica e porta, ao estudo com ultrassom doppler: o grupo de estudo (inversão - diâmetro da veia esplênica igual ou maior que o da veia porta) e o grupo controle (diâmetro da veia porta maior que o da veia esplênica). Foram identificados 21 pacientes que apresentavam inversão do calibre portoesplênica (calibre da veia esplênica maior ou igual ao calibre da veia porta).

O tratamento cirúrgico da hipertensão portal esquistossomótica seguiu a padronização do serviço de cirurgia geral do Hospital das Clínicas da UFPE, com esplenectomia + desvascularização da grande curvatura gástrica + ligadura da veia gástrica esquerda + biópsia hepática + esclerose endoscópica pós-operatória das varizes esofágicas ${ }^{3,17,18,20}$. Quando da presença de varizes de fundo gástrico (64/169) foi associado ao procedimento ci- rúrgico, a abertura do estômago e sutura das varizes de fundo gástrico como rotina.

A esplenectomia segue uma padronização que inclui a abertura da retrocavidade, abordagem e ligadura da artéria esplênica próximo ao hilo esplênico, liberação do polo inferior do baço, ligadura dos vasos no ligamento gastroesplênico, liberação posterior do baço e divisão dos ligamentos esplênicos, e abordagem do pedículo com dupla ligadura e secção da veia esplênica e nova ligadura e secção da artéria esplênica.

Todos os pacientes foram avaliados no pós-operatório no ambulatório de Hipertensão portal e Fígado do Hospital das Clínicas para o seguimento, com realização e registro de exames clínicos, laboratoriais, endoscópicos e ultrassonográficos, acrescentando de rotina a ultrassonografia doppler de vasos portais, para o registro das características pós-operatórias do fluxo portal.

O seguimento médio foi de 28,5 meses no grupo controle e 23,6 meses no grupo de estudo.

Todos os pacientes apresentavam epidemiologia positiva para a esquistossomose mansônica, caracterizada por contato com águas paradas em área endêmica, bem como antecedentes de hemorragia digestiva alta, manifestada por hematêmese elou por melena.

Os critérios de inclusão foram: antecedente de hemorragia digestiva alta;varizes esofagianas ao exame endoscópico; atividade enzimática não inferior à 50\%; sorologia viral para hepatite B ou C negativos; confirmação, na biopsia hepática, de doença esquistossomótica pura.

Os critérios de exclusão foram: hepatopatia mista; hemorragia digestiva não-varicosa.

A idade dos pacientes variou entre 22 e 56 anos, com uma média de 40,9 anos. e foram operados de maneira eletiva, fora do quadro agudo de hemorragia digestiva.

A classificação do grau de fibrose esquistossomótica obedeceu aos critérios descritos por Coelho ${ }^{23}$. De acordo com a sua intensidade o grau de fibrose foi classificado em: Grau I - os espaços porta apresentam-se com maior riqueza de células conjuntivas jovens, discreta produção de colágeno e presença variável de infiltrado inflamatório. A lâmina periportal e o retículo são normais; Grau II - há expansão do tecido conjuntivo periportal com emissão de septos colágenos radiais, conferindo ao mesmo um aspecto estrelado; Grau III - os septos conjuntivos formam pontes com outros espaços porta ou com as veias hepáticas, havendo neoformação angiomatóide bem evidente.

Os critérios diagnósticos do hiperesplenismo secundário à hipertensão portal esquistossomótica foram: 1) anemia (hemoglobina menor que $13 \mathrm{~g} / \mathrm{dl}$ nos homens e menor que $12 \mathrm{~g} / \mathrm{dl}$ nas mulheres), leucopenia (menos de 4000 leucócitos $/ \mathrm{mm}^{3}$ ), trombocitopenia (menos de 150.000 plaquetas $/ \mathrm{mm}^{3}$ ) ou a combinação destes; 2) esplenomegalia; 3 ) melhora ou regressão após a esplenectomia ${ }^{15}$. 


\section{RESULTADOS}

O grupo controle e grupo de estudo (inversão) foram constituídos por 148 e 21 pacientes, respectivamente. O seguimento médio pós-operatório foi de 28,5 meses para os pacientes do Grupo controle e 23,6 meses para os pacientes do Grupo de inversão. A permanência hospitalar foi de 7,7 e 6,1 dias os pacientes dos Grupos controle e inversão, respectivamente. A ocorrência de varizes de fundo gástrico nos pacientes do Grupo controle foi de 38,5\% (57\148) e de 33,3\% (7121) nos pacientes do grupo de inversão.

A média dos calibres da veia porta no pré-operatório dos grupos controle e inversão foram de $1,49 \mathrm{~cm}$ e $0,98 \mathrm{~cm}(p<0,05)$, respectivamente, enquanto a média dos calibres da veia esplênica no pré-operatório dos grupos controle e inversão foram de 1,14 e 1,07 ( $p>0,05)$.

A graduação da fibrose periportal foi assim distribuída no grupo controle: grau I = 17,6\% (26\148), grau II $=41,9 \%(62 / 148)$ e grau III $=40,5 \%(60 / 148)$, enquanto no grupo de inversão: grau I $=28,6 \%(6 / 21)$, grau $\mathrm{II}=33,3 \%(7 / 21)$ e grau $\mathrm{III}=38,1 \%(8 / 21)$, sem qualquer diferença estatística entre essas classes ( $p>0,05)$. A necessidade de hemotransfusão foi de 35,2\% e $33,3 \%$, com média de $2,45 \mathrm{CH}$ e $1,57 \mathrm{CH}$, nos grupos de controle e inversão, respectivamente $(p>0,05)$. A média do peso do baço foi de 1043,6g (347 - 6000g) no grupo controle e $859,4 \mathrm{~g}(500-1300 \mathrm{~g})$ no grupo de inversão $(p>0,05)$. Quanto ao calibre das varizes esofagianas, houve a seguinte distribuição no grupo controle: Grosso (37\% - 17\46); Médio (17,4\% - 8\46); Fino (10,9\% - 5146); Fino-médio (4,3\% - 2\46); Médio-grosso (30,4\% - 14\46). No grupo de inversão: Grosso (45,4\% - 5\11); Fino e Médio (36,4\% - 4111); Médio e Grosso (18,2 - 2\11), sem diferença estatística entre essas classes $(p>0,05)$. (Tabela 1)

Quanto aos dados laboratoriais, não houve diferença estatística entre os níveis de hemoglobina, leucócitos, funções renal e hepática (Tabela 2). Entretanto, houve significância estatística $(p<0,05)$ na diferença da média dos níveis séricos de plaquetas entre o grupo controle (106.647 $\mathrm{Mmm} 3)$ e o grupo de inversão (65.950 Imm3).

No que se refere às complicações pós-operatórias, houve no grupo controle: trombose portal em 16,9\% (11/65); ressangramento em 13,4\% (13/97), com mortalidade de $4,1 \%$. No grupo da inversão portolesplênica, identificamos ressangramento em $23,1 \%(3 \backslash 13)(p>0,05)$ e ausência de trombose portal $(p<0,05)$, com mortalidade de $4,8 \%(p>0,05)$.

Tabela 1- Relação entre inversão do calibre portolesplênico e os resultados cirúrgicos de pacientes portadores de Esquistossomose hepatoesplênica.

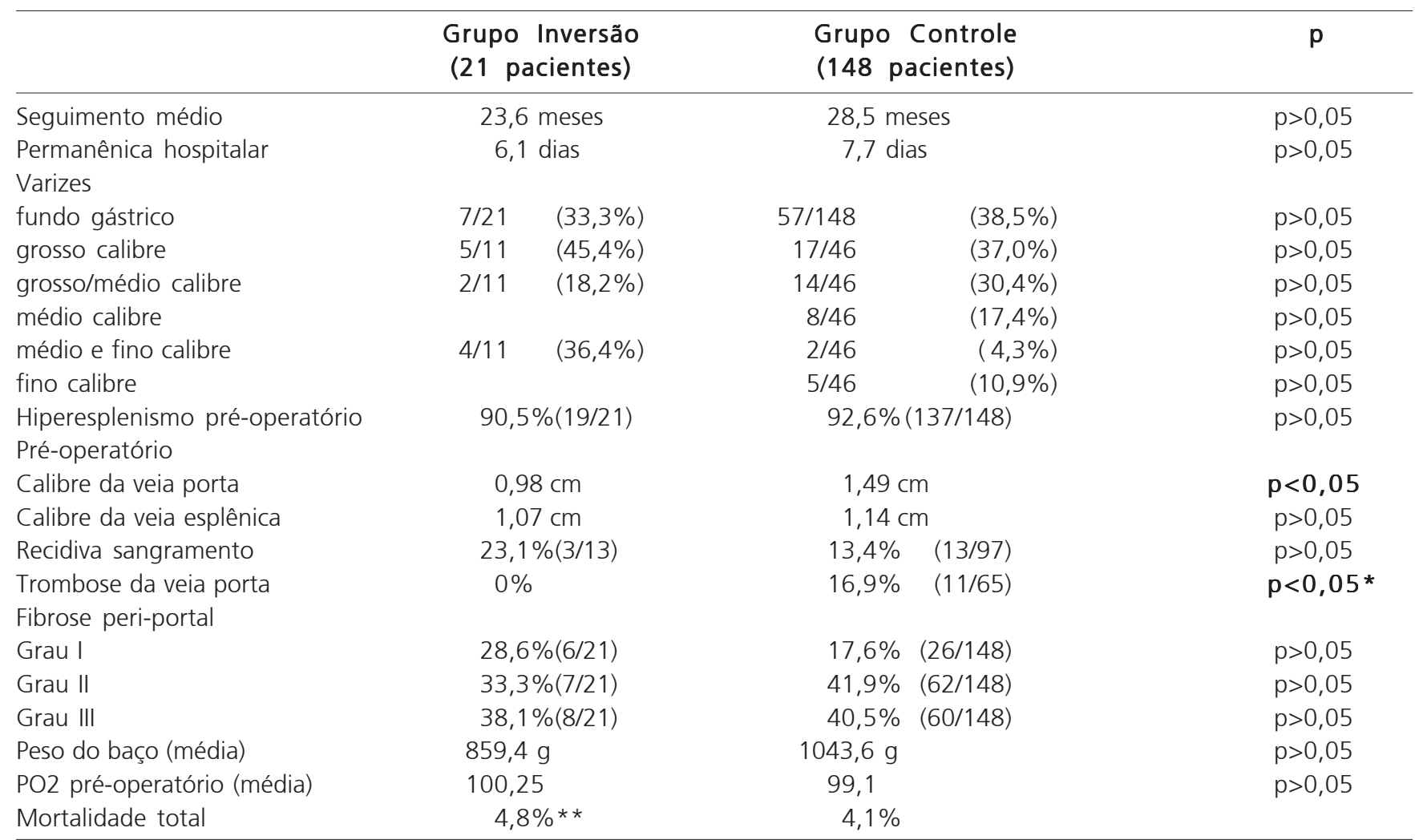

* - Teste exato de Fisher.

** - Um paciente foi a óbito por progressão de Linfoma não-Hodgkin. 
Tabela 2 - Relação entre inversão do calibre portolesplênico e os resultados laboratoriais pré-operatórios de pacientes portadores de Esquistossomose hepatoesplênica.

\begin{tabular}{|c|c|c|c|}
\hline & $\begin{array}{l}\text { Grupo Inversão } \\
\text { (21 pacientes) }\end{array}$ & $\begin{array}{l}\text { Grupo Controle } \\
\text { (148 pacientes) }\end{array}$ & $p$ \\
\hline Hemoglobina & $11,5 \mathrm{~g} \%$ & $10,34 \mathrm{~g} \%$ & $p>0,05$ \\
\hline Leucócitos & $4330 \backslash \mathrm{mm} 3$ & $4000 \backslash \mathrm{mm} 3$ & $p>0,05$ \\
\hline Plaquetas & $65.950 \backslash \mathrm{mm} 3$ & $106.647 \backslash \mathrm{mm} 3$ & $p<0,05$ \\
\hline Tempo de protombina & $15 s$ & $15 s$ & $p>0,05$ \\
\hline Glicose & $112,8 \mathrm{~g} \backslash d \mathrm{l}$ & 103,5 gldl & $p>0,05$ \\
\hline Uréia & $29,9 \mathrm{~g} \backslash \mathrm{dl}$ & $29,11 \mathrm{~g} \backslash \mathrm{dl}$ & $p>0,05$ \\
\hline Creatinina & 0,7 & $0,8 \mathrm{~g} \backslash \mathrm{dl}$ & $p>0,05$ \\
\hline Albumina & $3,34 \mathrm{gldl}$ & $3,51 \mathrm{~g} \backslash \mathrm{dl}$ & $p>0,05$ \\
\hline TGO & $46,7 \mathrm{~g} \backslash \mathrm{dl}$ & $39,5 \mathrm{~g} \backslash \mathrm{dl}$ & $p>0,05$ \\
\hline TGP & 49 gldl & $40,0 \mathrm{~g} \backslash \mathrm{dl}$ & $p>0,05$ \\
\hline Bilirrubinas totais & $1,11 \mathrm{~g} \backslash \mathrm{dl}$ & $0,96 \mathrm{~g} \backslash \mathrm{dl}$ & $p>0,05$ \\
\hline
\end{tabular}

\section{DISCUSSÃO}

A grande polêmica relacionada ao tratamento cirúrgico da hipertensão portal esquistossomótica reside no fato de que há maneiras diferentes de se abordar o problema. Na tentativa de se reduzir ao máximo a hemorragia varicosa, são realizados procedimentos que podem comprometer a função hepática e deteriorar em longo prazo o estado geral do paciente. Por outro lado, há procedimentos que tem pouca influência na função hepática, mas que cursam com uma recidiva hemorrágica um pouco mais elevada ${ }^{24}$.

Segundo Abrantes ${ }^{25}$, a operação ideal para tratar a hipertensão portal de origem esquistossomótica deveria prevenir a recidiva hemorrágica, não provocar encefalopatia, não agravar a função hepática e curar o hiperesplenismo.

Seguimos uma doutrina implantada e difundida pelo Prof. Salomão Kelner, a partir do início da década de $1960^{26}$. Esta doutrina se baseava em um tratamento cirúrgico que atendesse à redução da pressão no compartimento portal, associado ao mínimo de alterações fisiológicas e que fosse capaz de reverter as alterações decorrentes do hiperesplenismo. Ao longo de quatro décadas continuamos seguindo e defendendo estes preceitos, evidentemente adequando os pilares do pensamento às novas tecnologias que se tornaram acessíveis e a novos conhecimentos fisiopatológicos adquiridos. Deste modo, realizamos desde 1992 a esplenectomia com ligadura da veia gástrica esquerda associada à desvascularização parcial da grande curvatura do estômago e posterior tratamento endoscópico das varizes esofágicas ${ }^{1-3,17,18,20}$.

A esplenectomia na esquistossomose hepatoesplênica com antecedentes de hemorragia digestiva determina uma correção nos valores das alterações hematológicas (anemia, número de leucócitos, linfócitos e plaquetas), e uma redução média da pressão portal na ordem de $28 \%{ }^{15,27-29}$.
Na tentativa de se identificar de maneira préoperatória os pacientes que pouco se beneficiam do tratamento cirúrgico através da esplenectomia com ligadura da veia gástrica esquerda associada à desvascularização parcial da grande curvatura do estômago, foram analisados dados clínicos, laboratoriais e ultrassonográficos em 169 pacientes dos quais 21 apresentavam inversão do calibre portolesplênico. Destes, nenhum apresentou trombose portal na avaliação pósoperatória com Doppler de vasos portais. Neste grupo, a recidiva de sangramento por hemorragia digestiva alta ocorreu em 23,1\%.

No entanto, evidenciamos que: a permanência hospitalar foi estatisticamente semelhante nos pacientes em que o calibre da veia porta era menor ou maior do que o calibre da veia esplênica; o hiperesplenismo não tem relação direta com a relação do calibre portolesplenica, estando presente em $90,5 \%$ dos pacientes do grupo de estudo (inversão) e em 92,6\% dos pacientes do grupo de estudo ( $p>0,05)$; o calibre da veia porta também apresenta relação com o peso do baço, aumentando progressivamente à medida que o peso do baço aumenta, esse dado já foi previamente identificado em trabalho de nossa instituição, nos dados encontrados neste atual artigo houve diferença estatística entre os grupos de inversão e controle nas médias dos calibres da veia porta no pré-operatório $(p<0,05)$; não houve diferença estatística quanto à recidiva do sangramento no grupo de pacientes com inversão porto/esplênica $(23,1 \%)$, enquanto no grupo controle, houve $13,4 \%(p>0,05)$.

Não observamos no grupo de inversão a presença de trombose portal, enquanto no grupo controle, a incidência foi de $16,9 \%(p<0,05)$. Ainda que diante de número restrito de pacientes no grupo de inversão, já podemos ter a indicação de que a trombose portal não representa uma complicação importante nesse grupo de pacientes. 
A mortalidade no período de pós-operatório não apresentou diferença entre os grupos de inversão e controle, com índices de 4,8\% e 4,1\% (p>0,05), respectivamente.

Não identificamos nenhuma alteração nos valores pré-operatórios de hemograma, glicose, uréia, creatinina, albumina, transaminases e nas bilirrubinas, entretanto, houve diferença estatística entre as médias dos níveis séricos de plaquetas entre os grupos de inversão e controle $(65.950 \mathrm{Imm} 3 \times 106.647 \mathrm{Imm3}-p<0,05)$, respectivamente.
No que diz respeito à comparação dos nossos dados com a literatura, não identificamos abordagens que discutissem a inversão do calibre das veias porta e esplênica nos resultados cirúrgicos dos pacientes portadores de esquistossomose hepatoesplênica.

Os resultados obtidos nas condições deste estudo nos permitem inferir que os pacientes com o calibre da veia esplênica maior ou igual ao calibre da veia porta não representam um grupo de pacientes com um risco elevado para complicações no tratamento cirúrgico da hipertensão portal esquistossomótica.

\title{
A $B$ B S T $R$ R A C
}

\begin{abstract}
Objective: To evaluate the morbidity and mortality in surgical treatment of schistosomal portal hypertension in patients with inversion of the Portal/Splenic Vein diameter ratio. Methods: We conducted a retrospective cross-sectional study of patients undergoing surgical treatment of portal hypertension in the period between September 1993 and January 2004. The study population was divided into two groups: a) Inversion - splenic vein diameter greater than or equal to portal vein's - and b) control group (portal vein diameter greater than the splenic vein's). Statistical comparisons used the Student $t$ test for averages difference, chi-square test for proportions difference and Fisher's exact test for small samples. Results: 169 patients were analyzed, with follow-up averaging 23.6 months. Twenty-one patients (12.4\%) had splenic vein caliber greater of equal than the portal vein's (Inversion - study group). The mean preoperative diameter of the portal and splenic veins were respectively 1.49 and $1.14 \mathrm{~cm}$ in the control group, and 0.98 versus $1.07 \mathrm{~cm}$ in the inversion group. The portal vein diameter was significantly higher in the control group when compared to the inversion group ( $p<0.05$ ). Varices in the gastric fundus were found in $33.3 \%$ of the inversion group and in $38.5 \%$ of patients in the control group. Postoperative rebleeding occurred in $23.1 \%$ of patients in the inversion group and in $13.4 \%$ of the control group ones ( $p>0.05)$. In the postoperative evaluation with Doppler ultrasonography of portal vessels, no cases of portal vein thrombosis were observed in the inversion group, whilst in the control group portal thrombosis was identified in $16.9 \%$ of the patients $(p<0.05)$. Death occurred in one $(4.8 \%)$ individual from the inversion group; mortality was $4.1 \%$ in the control group $(p>0.05)$. The mean serum level of platelets was significantly lower $(65,950 / \mathrm{mm} 3)$ in the inversion group than in the controls $(106,647 / \mathrm{mm} 3)(p<0.05)$. Conclusion: The results suggest that the reversal of portal/splenic vein caliber ratio does not represent a contraindication to surgical treatment of schistosomal portal hypertension.
\end{abstract}

Key words: Schistosomiasis. Splenectomy. Risk factors. Portal vein.Hypertension, portal.

\section{REFERENCIAS}

1. Ferraz AAB, Lopes EPA, Bacelar TS, Marcello JC, Silva LMM, Ferraz EM. Tratamento cirúrgico da hipertensão portal esquistossomótica no HC-UFPE: análise de 131 casos. Rev Col Bras Cir 2000;27(5):332-

2. Ferraz EM, Bacelar TS, Ferraz AAB, Pagnossin G, Henriques Filho GATM, Mariz FEN. Infecção de ferida pós-esplenectomia em pacientes com ou sem esquistossomose hepatoesplênica. Rev Col Bras Cir 1991,18(3):75-9.

3. Ferraz AA, Lopes EP, Barros FM, Sette MJ, Arruda SM, Ferraz EM. Splenectomy plus left gastric vein ligature and devascularization of the great curvature of the stomach in the treatment of hepatosplenic schistosomiasis. Postoperative endoscopic sclerosis is necessary? Arq Gastroenterol 2001; 38(2):84-8.

4. Ferraz AA, Wanderley GJ, Santos MA Jr, Mathias CA, Araújo JG Jr, Ferraz EM. Effects of propranolol on human postoperative ileus. Dig Surg 2001;18(4):305-10.

5. Makdissi FF, Herman P, Pugliese V, de Cleva R, Saad WA, Cecconello I, D'Albuquerque LA. Long-term results of esophagogastric devascularization and splenectomy associated with endoscopic treatment in schistosomal portal hypertension. World J Surg 2010;34(11):2682-8

6. Makdissi FF, Herman P, Machado MA, Pugliese V, D'Albuquerque LA, Saad WA. Portal vein thrombosis after esophagogastric devascularization and splenectomy in schistosomal portal hypertension patients: what's the real importance? Arq Gastroenterol 2009:46(1):50-6.

7. de Cleva R, Herman P, D'albuquerque LA, Pugliese V, Santarem $\mathrm{OL}$, Saad WA. Pre- and postoperative systemic hemodynamic evaluation in patients subjected to esophagogastric devascularization plus splenectomy and distal splenorenal shunt: a comparative study in schistomomal portal hypertension. World J Gastroenterol 2007;13(41):5471-5.

8. Macêdo LG, Lopes EP, de Albuquerque Mde F, Markman-Filho B, Véras $\mathrm{FH}$, de Araújo AC, Ferraz AA. Occurrence of hepatopulmonary syndrome in patients with cirrhosis who are candidates for liver transplantation. J Bras Pneumol 2010;36(4):432-40.

9. de Cleva R, Herman P, Saad WA, Pugliese V, Zilberstein B, Rodrigues $\mathrm{JJ}$, Laudanna AA. Postoperative portal vein thrombosis in patients with hepatosplenic mansonic schistosomiasis: relationship with intraoperative portal pressure and flow. A prospective study. Hepatogastroenterology 2005;52(65):1529-33.

10. Cleva R, Saad WA, Herman P, Pugliese V, Zilberstein B, Laudanna AA, Gama-Rodrigues JJ. Portal hyperflow in patients with hepatosplenic mansonic schistosomiasis. Rev Hosp Clin Fac Med Sao Paulo 2004;59(1):10-4.

11. Aufses AH Jr, Schaffner F, Rosenthal WS, Herman BE. Portal venous pressure in "pipestem" fibrosis of the liver due to schistosomiasis. Am J Med 1959:27:807-10.

12. Siqueira LT, Ferraz AA, Campos JM, De Lima Filho JL, Albuquerque MC, de Lima-Aires A, Ribeiro MH, Cavalcanti MT, De Lima BCC, 
Ferraz EM. Analysis of plasma citrulline and intestinal morphometry in mice with hepatosplenic schistosomiasis. Surg Infect (Larchmt) 2010;11(5):419-26.

13. Ferreira FG, Forte WC, Assef JC, De Capua A Jr. Effect of esophagogastric devascularization with splenectomy on schistossomal portal hypertension patients' immunity. Arq Gastroenterol 2007;44(1):44-8.

14. Assef JC, de Capua Jr A, Szutan LA. Treatment of recurrent hemorrhage esophageal varices in schistosomotic patients after surgery. Rev Assoc Med Bras 2003;49(4):406-12.

15. Maia MD, Lopes EP, Ferraz AA, Barros FM, Domingues AL, Ferraz EM. Evaluation of splenomegaly in the hepatosplenic form of mansonic schistosomiasis. Acta Trop 2007;101(3):183-6.

16. Ferraz AA, Campos JM, Júnior JG, De Albuquerque AC, Ferraz EM. Gut bacterial translocation and postoperative infections: a prospective study in schistosomotic patients. Surg Infect (Larchmt) 2005;6(2):197-201.

17. Ferraz AA, de Albuquerque PC, Lopes EP, de Araújo JG Jr, CarvaIho $\mathrm{AH}$, Ferraz EM. The influence of periportal (pipestem) fibrosis on long term results of surgical treatment for schistosomotic portal hypertension. Arq Gastroenterol 2003;40(1):4-10.

18. Ferraz AA, Bacelar TS, Silveira MJ, Coelho AR, Câmara Neto RD, de Araújo Júnior JG, Ferraz EM. Surgical treatment of schistosomal portal hypertension. Int Surg 2001;86(1):1-8.

19. Petroianu A, Resende V, Da Silva RG. Late follow-up of patients submitted to subtotal splenectomy. Int J Surg 2006;4(3):172-8.

20. Ferraz AAB, Lopes EPA, Araújo Júnior JGC, Lima BA, Cantarelli F, Ferraz EM. Varizes de fundo gástrico na hipertensão portal esquistossomótica: resultados cirúrgicos. Rev Col Bras Cir 2003;30(1):21-8.

21. Ferreira FG, Ribeiro MA, de Fátima-Santos M, Assef JC, Szutan LA. Doppler ultrasound could predict varices progression and rebleeding after portal hypertension surgery: lessons from 146 EGDS and 10 years of follow-up. World J Surg 2009;33(10):213643.

22. Carvalho DL, Capua A Jr, Leme PL. Portal flow and hepatic function after splenectomy and esophagogastric devascularization. Int Surg 2008;93(6):314-20.

23. Coelho RB. Anatomia patológica das afecções hepáticas. Recife: UFPE; 1971. Lesões hepáticas secundárias; p.59-77.
24. Ferraz EM, Ferraz AAB. Tratamento cirúrgico da hipertensão portal esquistossomótica. In: Malta J. Esquistossomose Mansônica. Recife: UFPE; 1994. p.235-49.

25. Abrantes WL. Análise crítica da cirurgia de hipertensão portal esquistossomótica. In: Oliveira-e-Silva A, D’Albuquerque LC. Hepatologia clínica e cirúrgica. São Paulo: Sarvier; 1986. p.67182.

26. Kelner S. Avaliação da esplenectomia e ligadura intra-esosafiana das varizes do esôfago na esquistossomose mansônica [tese para professor catedrático]. Recife: Universidade Federal do Recife, Faculdade de Medicina; 1965. 312p.

27. Silveira GK. Efeito da esplenectomia sobre o hormônio de crescimento em pacientes portadores de esquistossomose hepatoesplênica e hipodesenvolvimento somático [dissertação]. Pernambuco: Universidade Federal de Pernambuco: 1976. 85p.

28. Domingues ALC. Ultra-sonografia na esquistossomose mansônica hepato-esplênica: avaliação da intensidade da fibrose periportal e da hipertensão porta [tese]. Pernambuco: Universidade Federal de Pernambuco, Faculdade de Medicina; 1998. 99p.

29. Kelner S, Ferreira PR, Dantas A, Lima filho JF, Souza AP, Carreiro Júnior JC, Ferraz EM, Silveira M, Coelho AR, Câmara Neto RD, Domingues LA. Ligadura de varizes esôfago-gástricas na hipertensão porta esquistossomótica: evolução de 25 anos. Rev Col Bras Cir 1982;9(4):140-6.

Recebido em 10/12/2009

Aceito para publicação em 15/02/2010

Conflito de interesse: nenhum

Fonte de financiamento: nenhuma

\section{Como citar este artigo:}

Ferraz AAB, Campos JM, Araújo Júnior JGC, Carvalho MRC, Ribeiro Neto JP, Ferraz EM. Influência da inversão do diâmetro veia porta / veia esplênica nos resultados do tratamento cirúrgico da hipertensão portal esquistossomótica. Rev Col Bras Cir. [periódico na Internet] 2011; 38(1). Disponível em URL: http://www.scielo.br/rcbc

Endereço para correspondência:

Álvaro Antônio Bandeira Ferraz

E-mail: aabf@truenet.com.br 